\title{
Aryloxy Diester Phosphonamidate Prodrugs of Phosphoantigens (ProPAgens) as Potent Activators of $V_{\gamma} 9 / \mathrm{V} \delta 2 \mathrm{~T}$-Cell Immune Responses
}

Hachemi Kadri, ${ }^{a, \ddagger, \#}$ Taher E. Taher, ${ }^{b, c, \ddagger}$ Qin Xu, ${ }^{a, \ddagger}$ Maria Sharif, ${ }^{b, c, \ddagger}$ Elizabeth Ashby, ${ }^{b, c}$ Richard T. Bryan, ${ }^{d}$ Benjamin E. Willcox, ${ }^{* b, c}$ Youcef Mehellou*a

\footnotetext{
a. School of Pharmacy and Pharmaceutical Sciences, Redwood Building, King Edward VII Avenue, Cardiff CF10 3NB, U.K.

b. Cancer Immunology and Immunotherapy Centre, University of Birmingham, Edgbaston, Birmingham B15 2TT, U.K.

c. Institute of Immunology and Immunotherapy, University of Birmingham, Edgbaston, Birmingham B15 2TT, U.K.

d. Institute of Cancer and Genomic Sciences, University of Birmingham, Edgbaston, Birmingham B15 2TT, U.K.

\#Current address: School of Chemistry, South Road, Durham University, Durham DH1 3LE, U.K.
}

\section{SUPPORTING FIGURES}

Serum stability of HMBP ProPAgen $\mathbf{4 d}$.

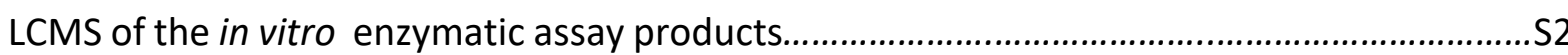

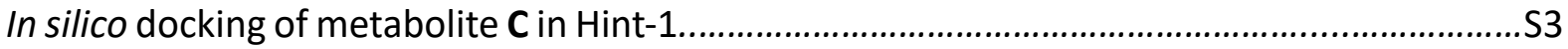

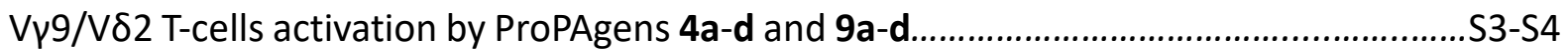

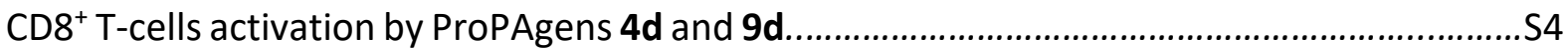

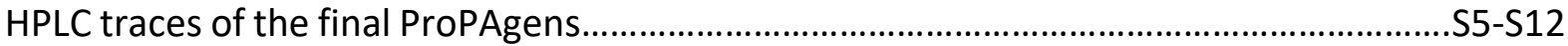

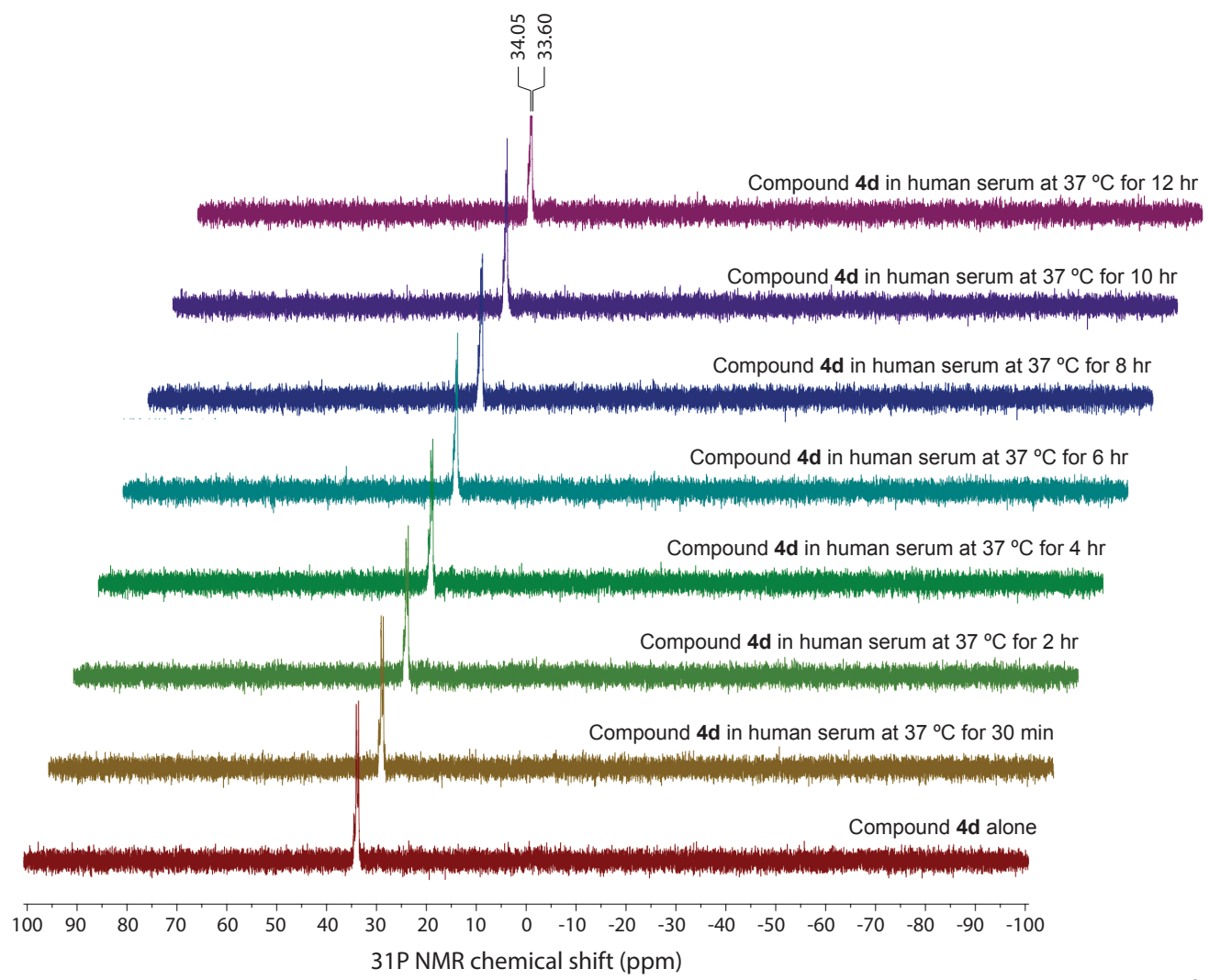

Figure S1. Stability of HMBP phosphonate ProPAgen $\mathbf{4 d}$ in human serum at $37^{\circ} \mathrm{C}$ for $12 \mathrm{~h}$ as monitored by ${ }^{31} \mathrm{P}$ NMR. 
A.

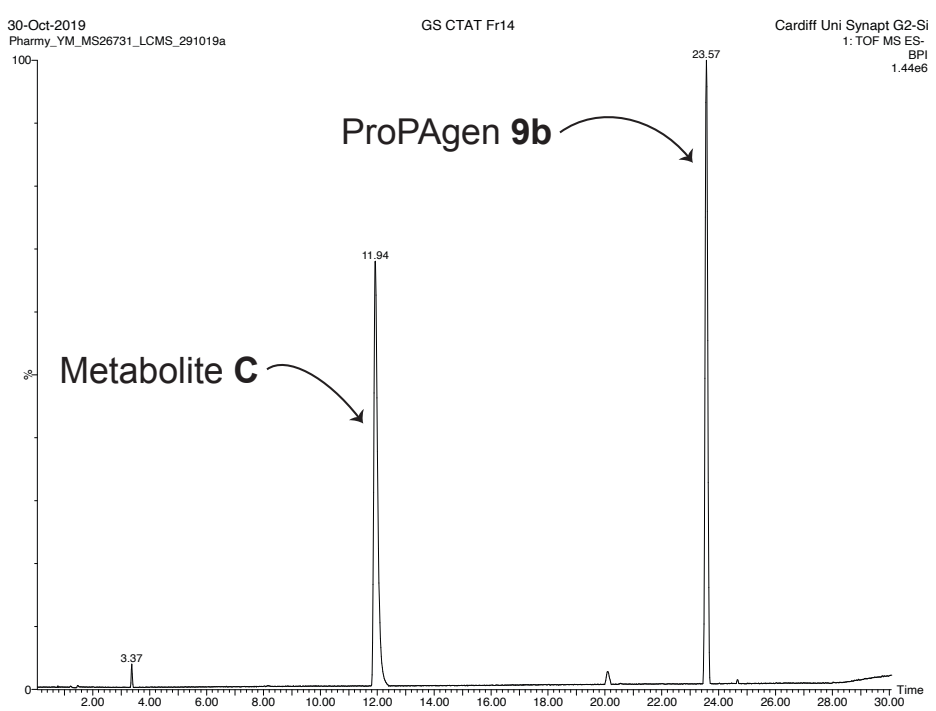

B.

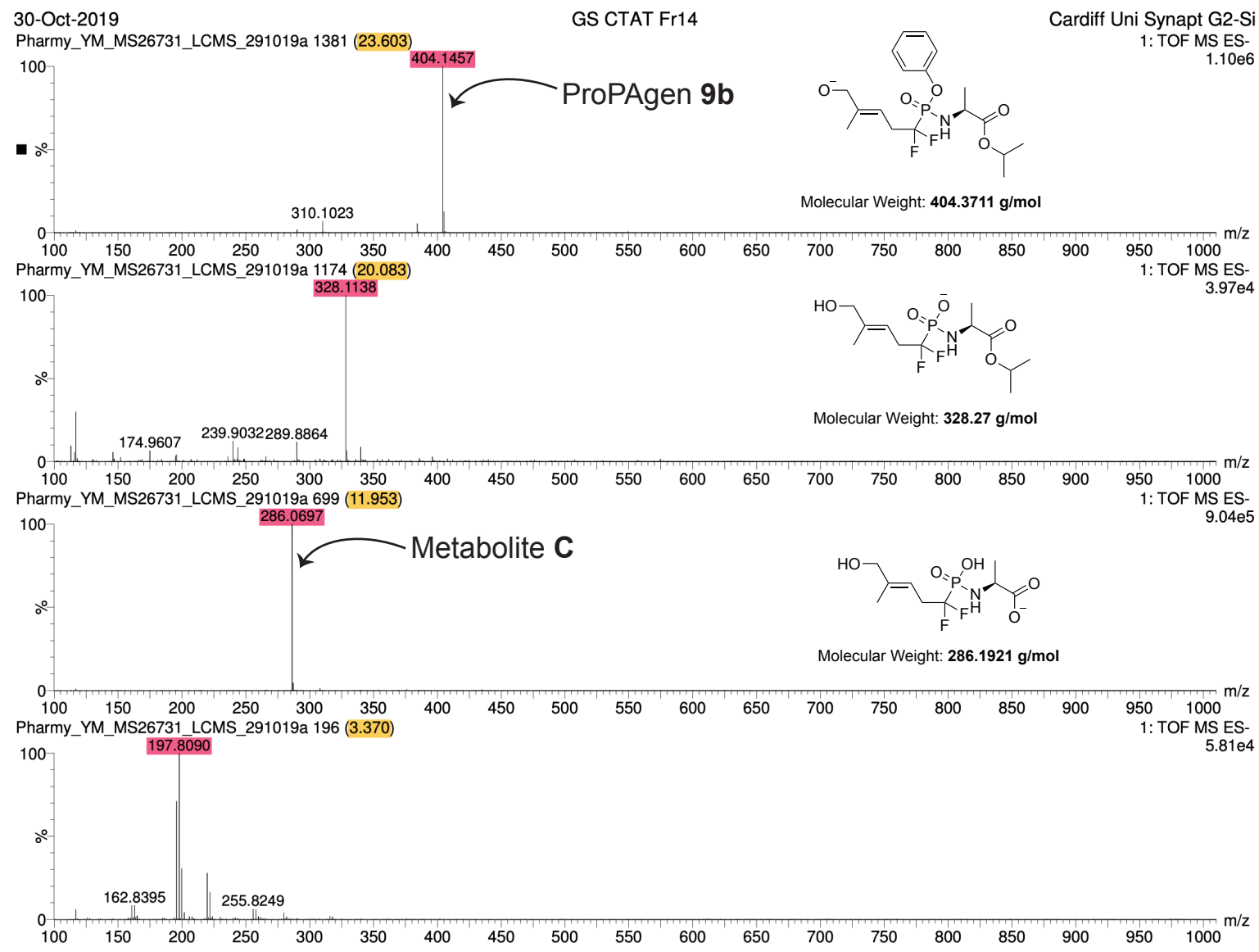

Figure S2. LC-MS analysis of the products of the carboxypeptidase Y-mediated metabolism of ProPagen 9b. A) HPLC analysis of the components of the metabolism products shows two major peaks with retention times of 11.94 and 23.57 minutes. Two minor peaks with retention times of 3.37 and 20.1 minutes were also detected. B) TOF MS ESanalysis of the two major peaks sowed that the product with a retention time of 23.57 minutes has a mass of 404.1457 $\mathrm{g} / \mathrm{mol}$, which corresponds to the mass of ProPAgen $9 \mathrm{~b}$, which has a calculated mass of $404.3711 \mathrm{~g} / \mathrm{mol}$ (-ve mode). The second major peak with a retention time of 11.94 minutes has a mass of $286.0697 \mathrm{~g} / \mathrm{mol}$, which corresponds to the calculated mass of metabolite $\mathbf{C}(286.1921 \mathrm{~g} / \mathrm{mol})$ (-ve mode). The two minor peaks with retention times 3.37 and 20.1 minutes have masses of 197.8090 and $328.1138 \mathrm{~g} / \mathrm{mol}$, with the latter one corresponding to ProPAgen $9 \mathrm{~b}$ without the phenyl group while the identity of the metabolite with retention time of 3.370 could not be identified. 


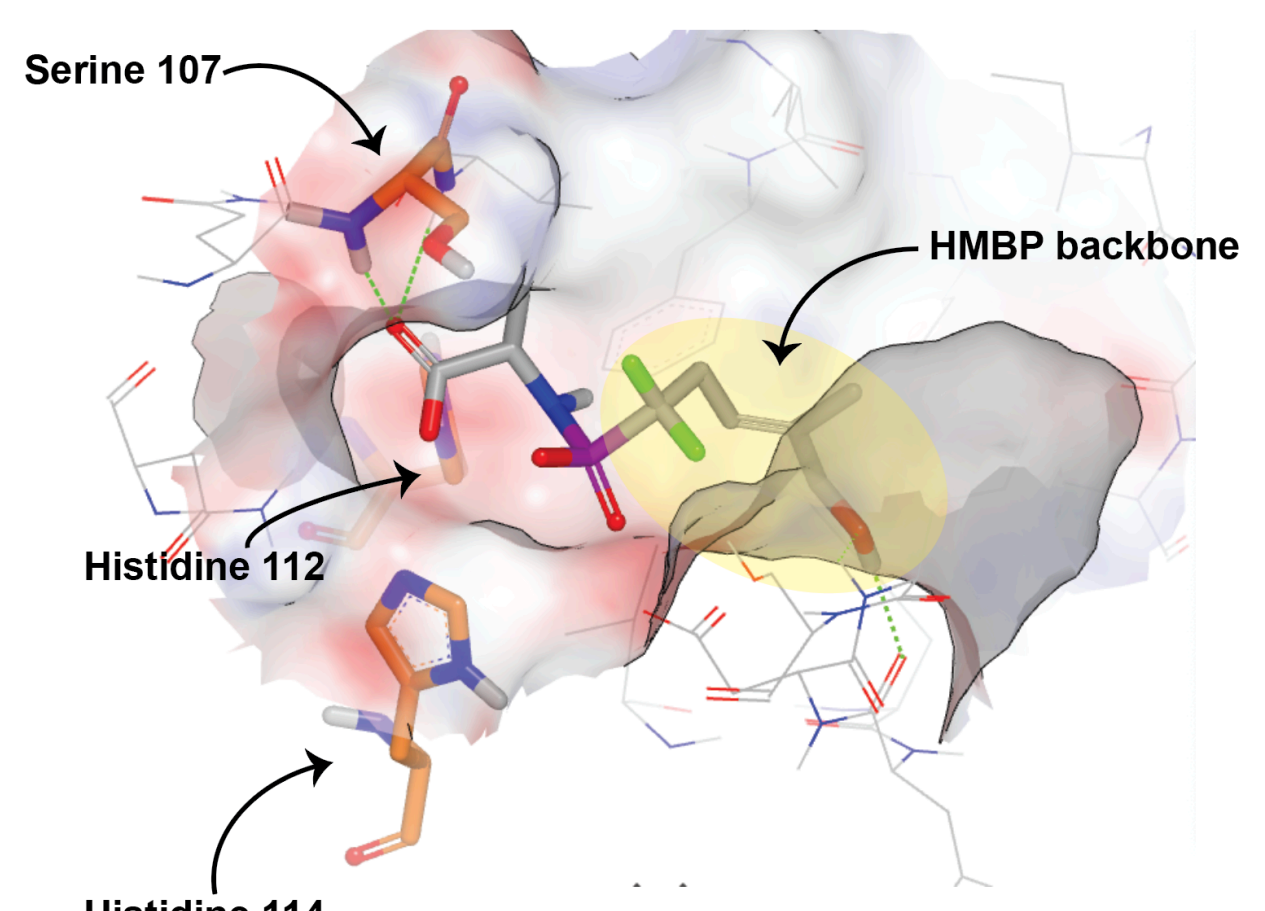

Histidine 114

Figure S3. Docking of metabolite $\mathbf{C}$ in the crystal structure of Hint-1 (PDB ID: 1KPF).
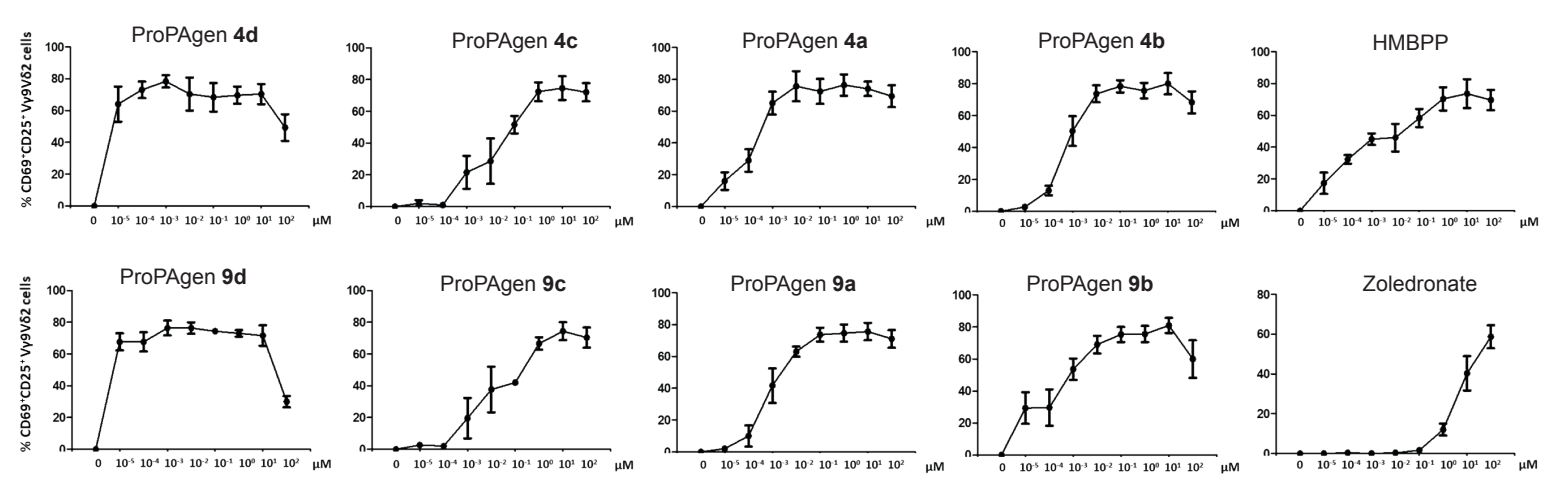

Figure S4. In vitro ProPAgen-mediated activation of $\mathrm{V} \gamma 9 / \mathrm{V} \delta 2$ T-cells following overnight incubation with HMBPP, zoledronate and HMBPP ProPAgens 4a-d (top panel) and 9a-d (top panel) at concentrations ranging from $10 \mathrm{pM}$ to $100 \mu \mathrm{M}$. Levels of activation are represented as $\%$ of $\mathrm{V} \gamma 9 / \mathrm{V} \delta 2 \mathrm{~T}$ cells that are CD69+ CD25+. Data are shown as mean $\pm \mathrm{SE}(n=4)$. 

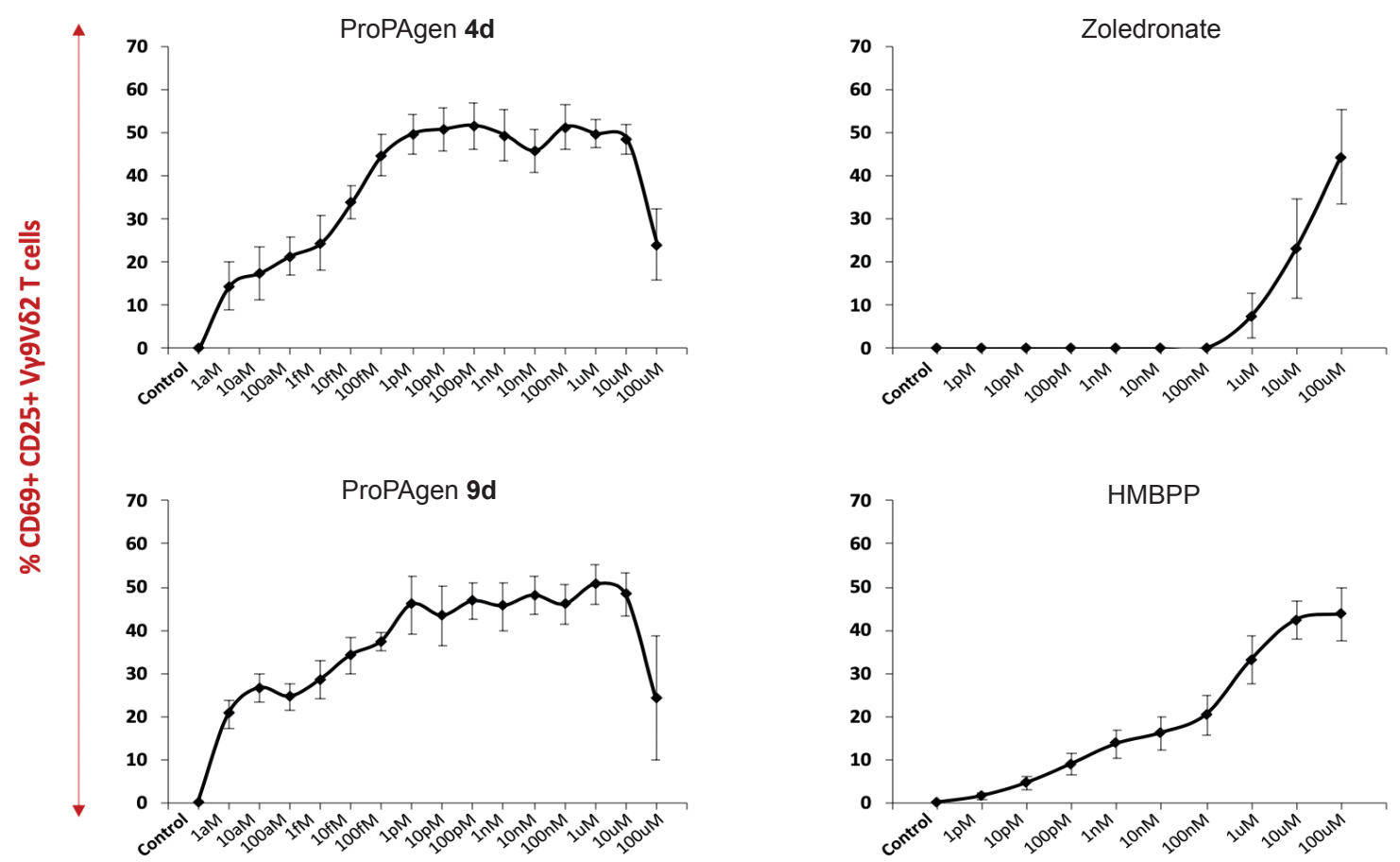

Figure S5. In vitro ProPAgen-mediated activation of $\mathrm{V} \gamma 9 / \mathrm{V} \delta 2$ T-cells following overnight incubation with HMBPP, zoledronate and HMBPP ProPAgens 4a-d (top panel) and 9a-d (top panel) at concentrations ranging from 1 aM to 100 $\mu \mathrm{M}$. Levels of activation are represented as $\%$ of $\mathrm{V} \gamma 9 / \mathrm{V} \delta 2 \mathrm{~T}$ cells that are CD69+ CD25+. Data is shown as mean $\pm \mathrm{SE}$ $(n=4)$.

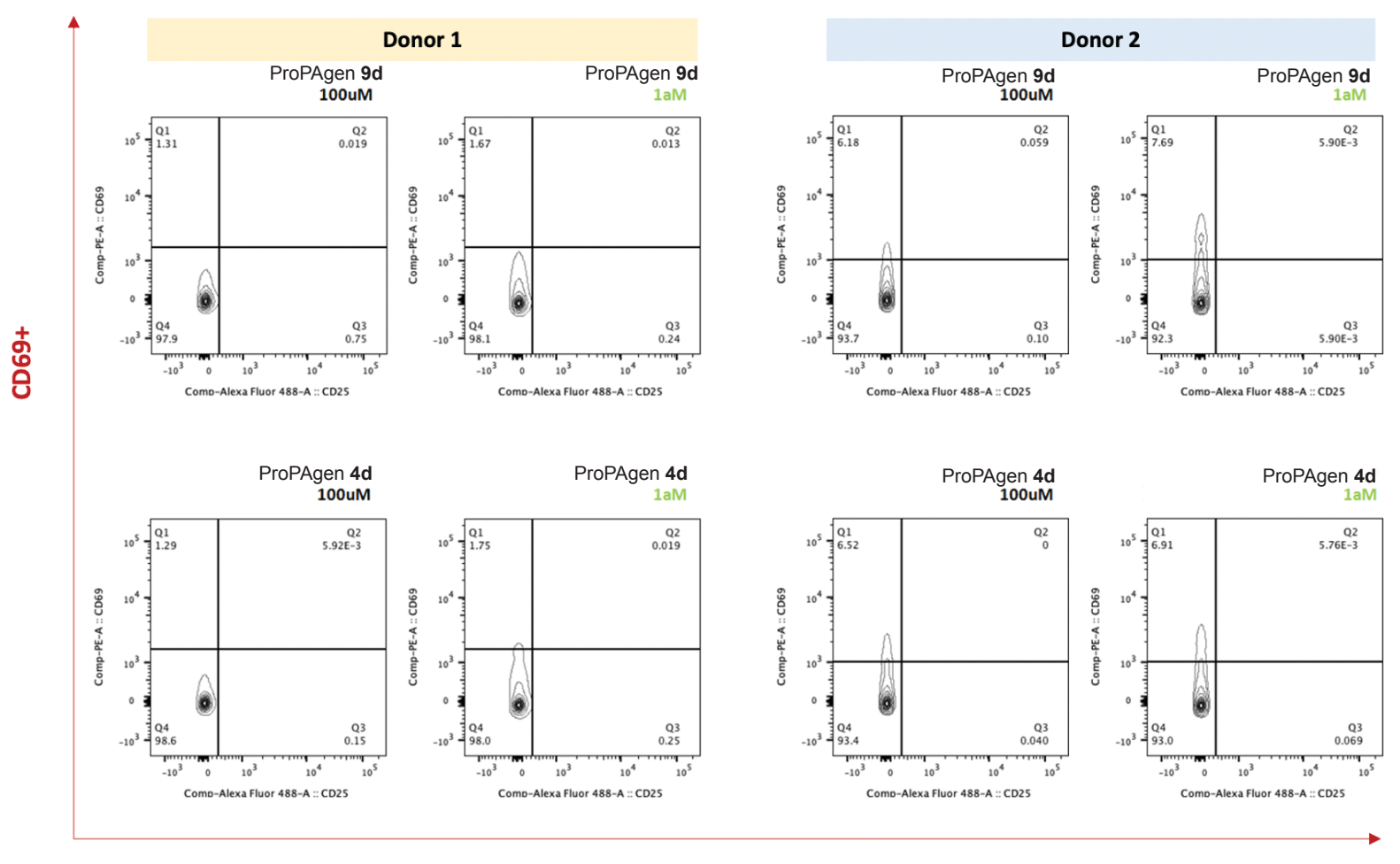

\section{CD25+}

Figure S6. $C D 8^{+} T$-cells are not activated by $V y 9 V \delta 2$-specific HMBP ProPAgens $\mathbf{4 d}$ and $\mathbf{9 d}$ at the two concentrations (1 $\mathrm{aM}$ and $100 \mu \mathrm{M}$ ) used. The data were obtained from PBMCs collected from two donors. 
ProPagen 4a:

08/11/2018 14:59:25 Page 1/1

\section{LabSolutions Analysis Report}

\section{<Sample Information>}

Sample Name : HK 91

Sample ID : HK $910.5 \mathrm{mlmin}$

Data Filename : 25102018 HK 91 001.Icd

Method Filename : method $0.5 \mathrm{mlmin} 251018 . \mathrm{lcm}$

Batch Filename : batch 2 251018.Icb

Vial \#

$1-3$

Vial \#

$\begin{array}{ll}\text { Injection Volume } & : 10 \mathrm{uL} \\ \text { Date Acquired } & : 25 / 10 / 2018 \text { 14:39:36 }\end{array}$

Date Processed $\quad:$ 25/10/2018 16:09:50

Sample Type : Unknown

Acquired by : : System Administrator

Processed by : System Administrator

\section{$<$ Chromatogram>}

$\mathrm{mV}$

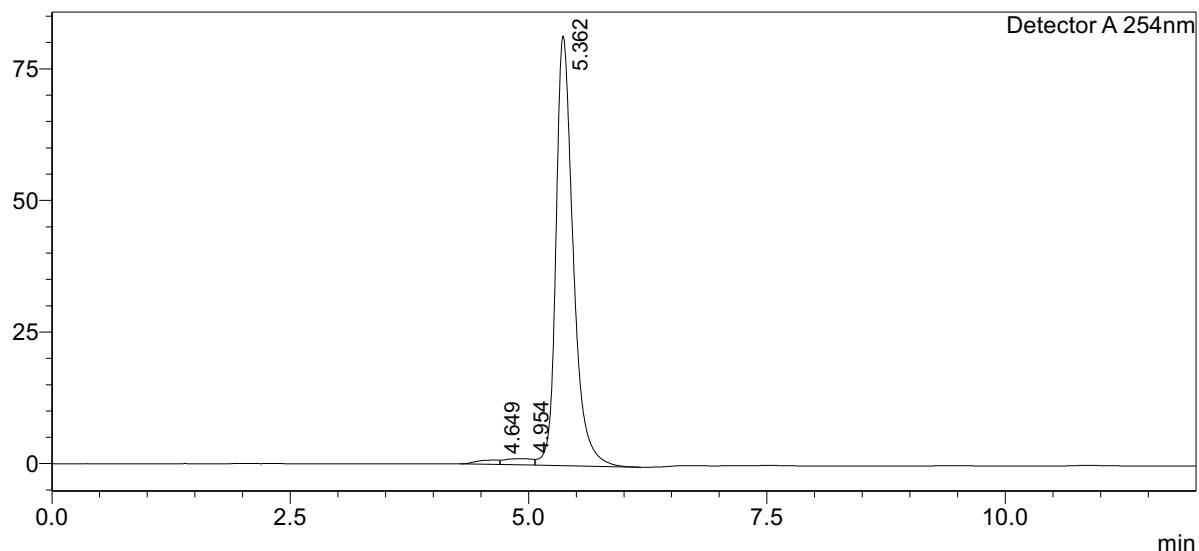

<Peak Table>

Detector A 254nm

\begin{tabular}{|r|r|r|r|r|}
\hline Peak\# & Ret. Time & \multicolumn{1}{|c|}{ Area } & Height & \multicolumn{1}{|c|}{ Area $\%$} \\
\hline 1 & 4.649 & 13088 & 823 & 1.225 \\
\hline 2 & 4.954 & 23889 & 1184 & 2.237 \\
\hline 3 & 5.362 & 1031137 & 81585 & 96.538 \\
\hline Total & & 1068113 & 83591 & 100.000 \\
\hline
\end{tabular}


ProPagen 4b:

04/12/2018 19:41:58 Page 1/1

\section{SHMADZu
LabSolutions Analysis Report}

\section{<Sample Information>}

Sample Name : HKP94

Sample ID : HKP94

Data Filename : HKP94-benzyl001.Icd

Method Filename : method 0.5mlmin90-20 041218.lcm

Batch Filename : HKP94rep.lcd.Icb

Vial \# : :1-1

Injection Volume : $5 \mathrm{uL}$

Date Acquired $\quad: 04 / 12 / 2018$ 18:45:25

Sample Type : Unknown

Date Processed : :04/12/2018 18:57:26

Acquired by : : System Administrator

Processed by : System Administrator

\section{$<$ Chromatogram>}

$\mathrm{mV}$

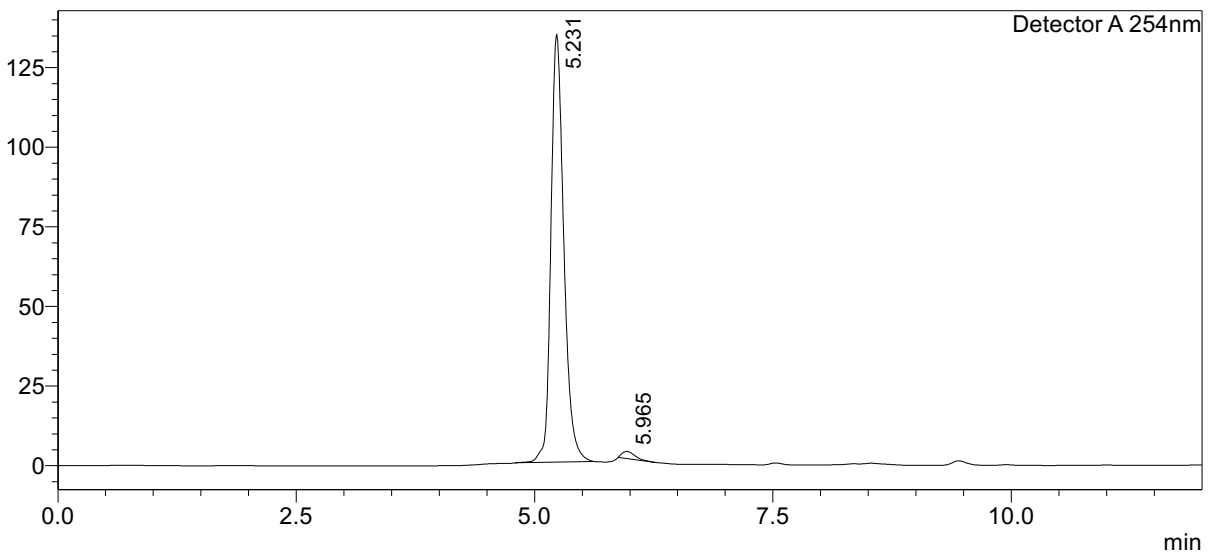

<Peak Table>

Detector A 254nm

\begin{tabular}{|c|c|c|c|c|}
\hline Peak\# & Ret. Time & Area & Height & Area $\%$ \\
\hline 1 & 5.231 & 1264426 & 134179 & 98.396 \\
\hline 2 & 5.965 & 20615 & 2209 & 1.604 \\
\hline Total & & 1285041 & 136388 & 100.000 \\
\hline
\end{tabular}




\section{ProPagen 4c:}

04/12/2018 17:47:22 Page 1/1

\section{LabSolutions Analysis Report}

\section{<Sample Information>}

Sample Name : HKP92

Sample ID : HKP92

Data Filename : HKP92-tertbutyl002.lcd

Method Filename : method 0.5mlmin90-20 041218.lcm

Batch Filename : HKtertButyl.Icb

Vial \# : : 1-1

Injection Volume : $10 \mathrm{uL}$

Sample Type : Unknown

Date Acquired $\quad: 04 / 12 / 201817: 26: 37$

Acquired by : : System Administrator

Date Processed : :04/12/2018 17:38:38

Processed by : : System Administrator

\section{$<$ Chromatogram $>$}

$\mathrm{mV}$

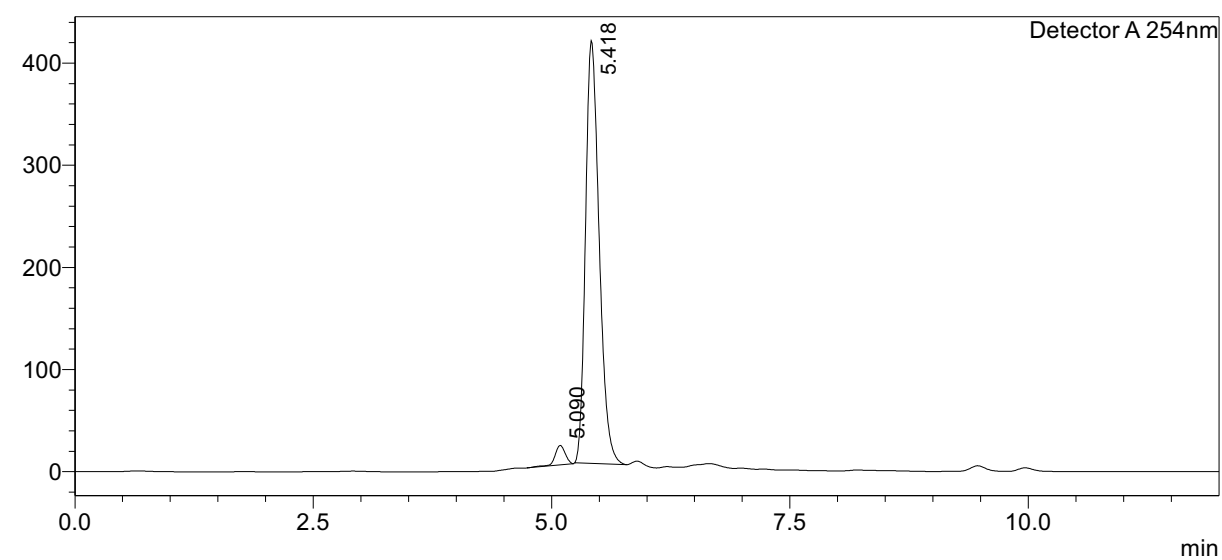

<Peak Table>

Detector A 254nm

\begin{tabular}{|c|c|c|c|c|}
\hline Peak\# & Ret. Time & Area & Height & Area\% \\
\hline 1 & 5.090 & 143836 & 19077 & 3.374 \\
\hline 2 & 5.418 & 4119042 & 413767 & 96.626 \\
\hline Total & & 4262878 & 432844 & 100.000 \\
\hline
\end{tabular}


ProPagen 4d:

05/12/2018 10:57:36 Page $1 / 1$

\section{SHMADZu
LabSolutions Analysis Report}

\section{<Sample Information>}

Sample Name : HKP89

Sample ID : HKP89

Data Filename : HKP89-benzyl006.Icd

Method Filename : method 0.5mlmin93-7 051218.lcm

Batch Filename : 97-3-5-12hk89benzyl.Icb

Vial \#

Injection Volume $: 5 \mathrm{uL}$

Date Acquired : :05/12/2018 10:36:49

Sample Type : Unknown

Date Processed : :05/12/2018 10:48:50

Acquired by : : System Administrator

Processed by : : System Administrator

\section{$<$ Chromatogram $>$}

$\mathrm{mV}$

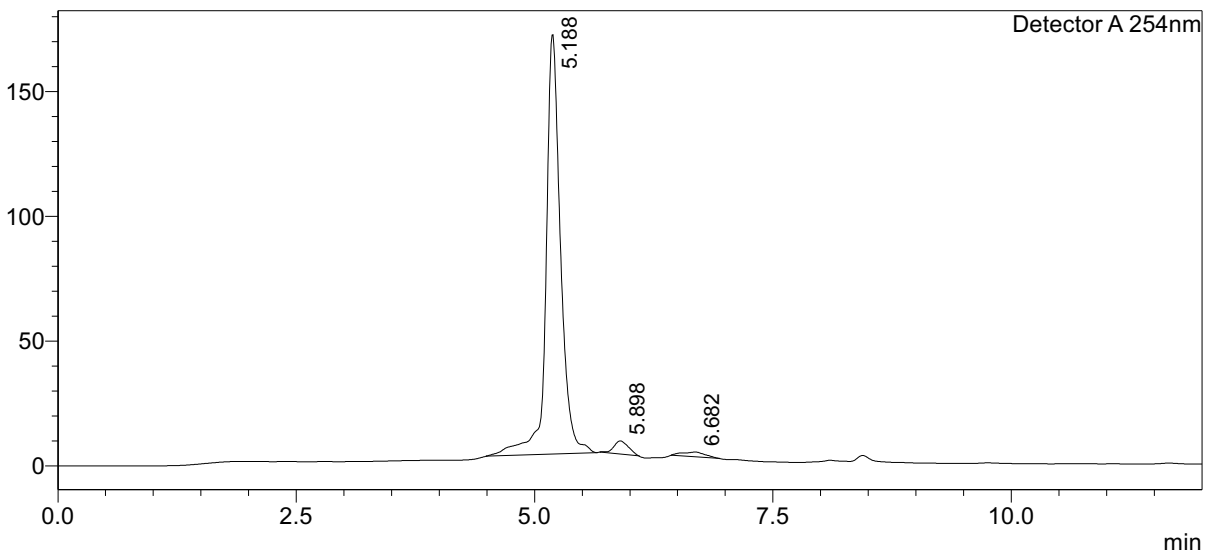

<Peak Table>

Detector A 254nm

\begin{tabular}{|r|r|r|r|r|}
\hline Peak\# & Ret. Time & \multicolumn{1}{|c|}{ Area } & Height & \multicolumn{1}{c|}{ Area\% } \\
\hline 1 & 5.188 & 1788024 & 167904 & 95.282 \\
\hline 2 & 5.898 & 56072 & 5257 & 2.988 \\
\hline 3 & 6.682 & 32466 & 1938 & 1.730 \\
\hline Total & & 1876562 & 175098 & 100.000 \\
\hline
\end{tabular}




\section{ProPagen 9a:}

\section{i.i. LabSolutions Analysis Report}

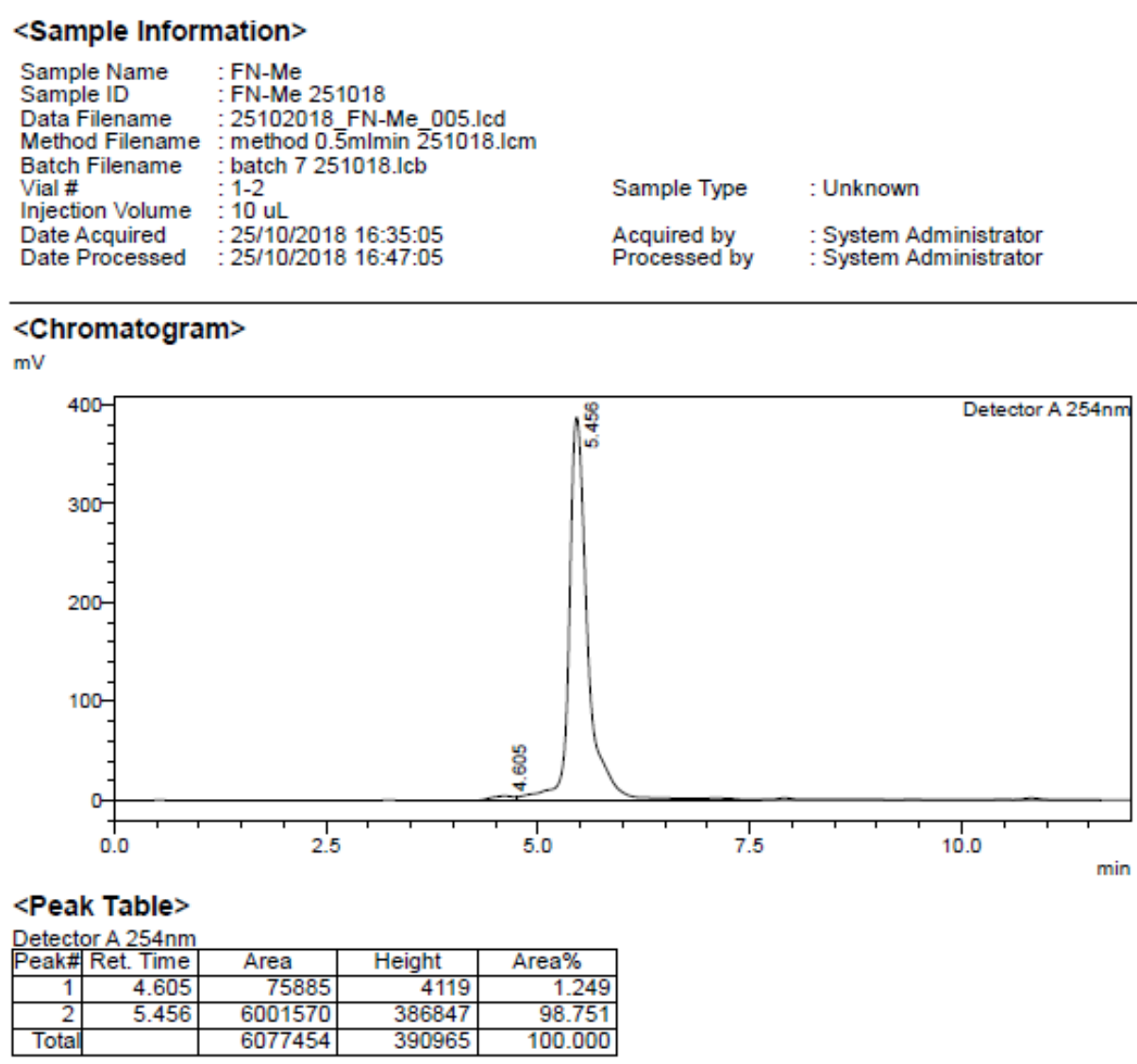




\section{ProPagen 9b:}

\section{iili LabSolutions Analysis Report}

\section{<Sample Information>}

\section{Sample Name : FN(ISO)}

Sample ID : FN(ISO1)001

Data Filename : $13112018 \mathrm{FN}($ ISO) 003.Icd

Method Filename : method $0 . \overline{5} \mathrm{mlmin} 251018 . \mathrm{Icm}$

Batch Filename : iso03 131118.Icb

Vial \# : $1-20$

: 20 uL

Date Acquired $\quad: 13 / 11 / 2018$ 12:33:24

Sample Type : Unknown

Date Processed $\quad: 13 / 11 / 2018$ 12:45:25

Acquired by : System Administrator Processed by : : System Administrator

$<$ Chromatogram $>$

$\mathrm{mV}$

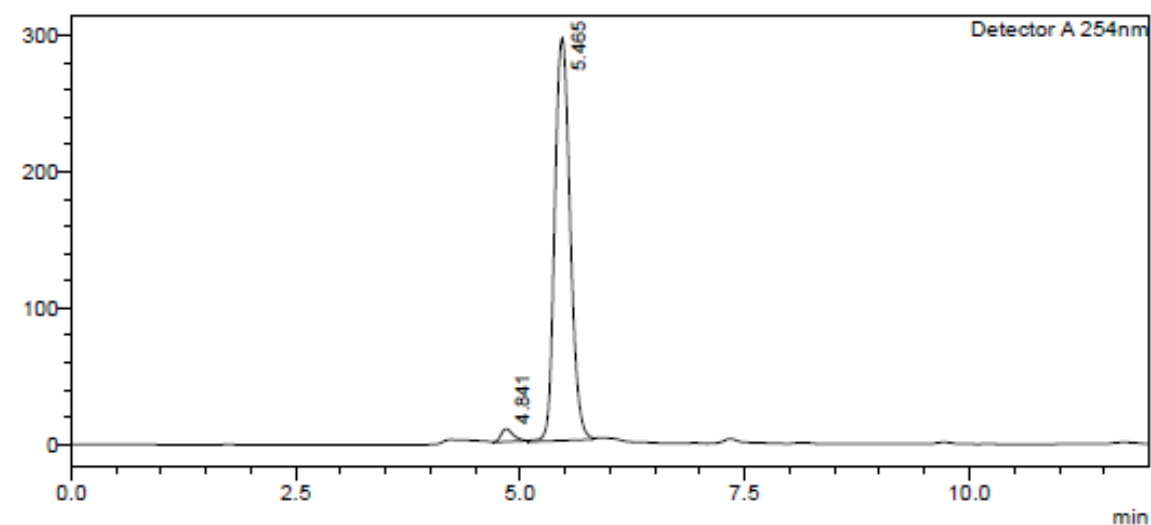

<Peak Table>

\begin{tabular}{|c|c|c|c|c|}
\hline Peak\# & Ret. Time & Area & Height & Area\% \\
\hline 1 & 4.841 & 87174 & 9386 & 2.41 \\
\hline 2 & 5.465 & 3515889 & 295057 & 97.58 \\
\hline & & 3603063 & 304442 & 100 \\
\hline
\end{tabular}




\section{ProPagen 9c:}

\section{2i:H LabSolutions Analysis Report}

\section{<Sample Information>}

Sample Name : XQ-tBu001

Sample ID : XQ-tBu001

Data Filename : XQ-tBu001.Icd

Method Filename : method $0.5 \mathrm{mlmin} 251018 . \mathrm{Icm}$

Batch Filename : tbu batch2 3-12-18.Icb

Batch Filename tou ba

Sample Type : Unknown

injection Volume : $10 \mathrm{uL}$

Date Acquired $\quad: 03 / 12 / 2018$ 13:16:46

Acquired by : System Administrator

Date Processed $\quad: 03 / 12 / 2018$ 13:28:47

$<$ Chromatogram $>$

$\mathrm{mV}$

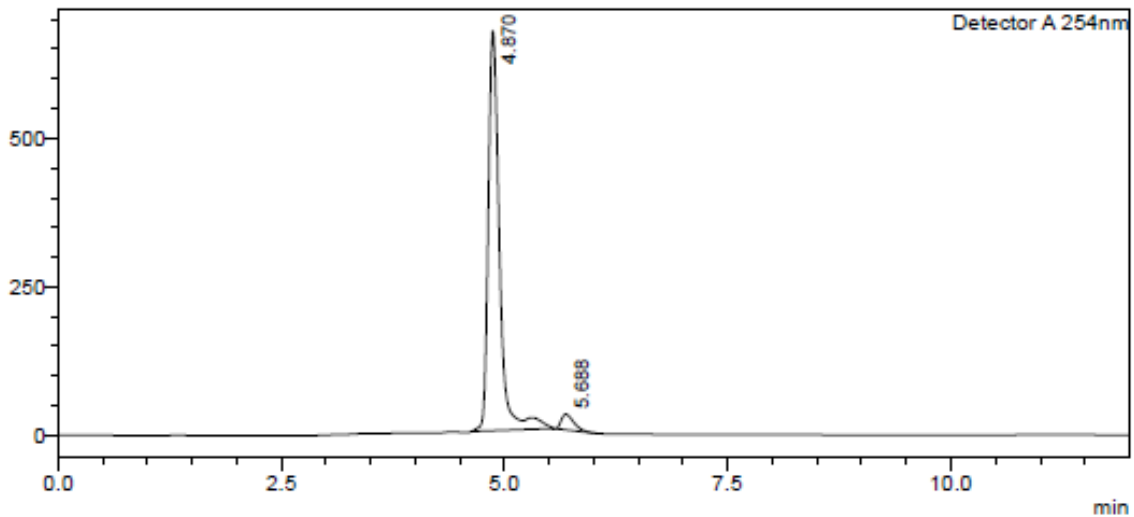

<Peak Table>

Detector A $254 \mathrm{~nm}$

\begin{tabular}{|r|r|r|r|r|}
\hline Peak\# & Ret. Time & \multicolumn{1}{|c|}{ Area } & Height & \multicolumn{1}{|c|}{ Area\% } \\
\hline 1 & 4.870 & 5800858 & 871680 & 05.808 \\
\hline 2 & 5.688 & 252997 & 26129 & 4.112 \\
\hline Total & & 6152855 & 697809 & 100.000 \\
\hline
\end{tabular}




\section{ProPagen 9d:}

\section{LabSolutions Analysis Report}

\section{<Sample Information>}

Sample Name : FN-Bn

Sample ID $\quad F N-B N-0.5 \mathrm{~m} / \mathrm{min}$

Data Filename :25102018 FN-Bn_001.Icd

Method Filename : method $0 . \overline{5} \mathrm{mlmin} \overline{2} 51018 . \mathrm{lcm}$

Batch Filename : batch $0.5 \mathrm{~m} / \mathrm{min} 251018 . \mathrm{lcb}$

$\begin{aligned} & \text { Vial \# } \\ & \text { Injection Volume }\end{aligned}: 10 \mathrm{uL}$

Date Acquired : : 25/10/2018 11:26:10

Sample Type : Unknown

Date Processed : :25/10/2018 16:11:17

Acquired by : System Administrator

Processed by : : System Administrator

$<$ Chromatogram $>$

$\mathrm{mV}$

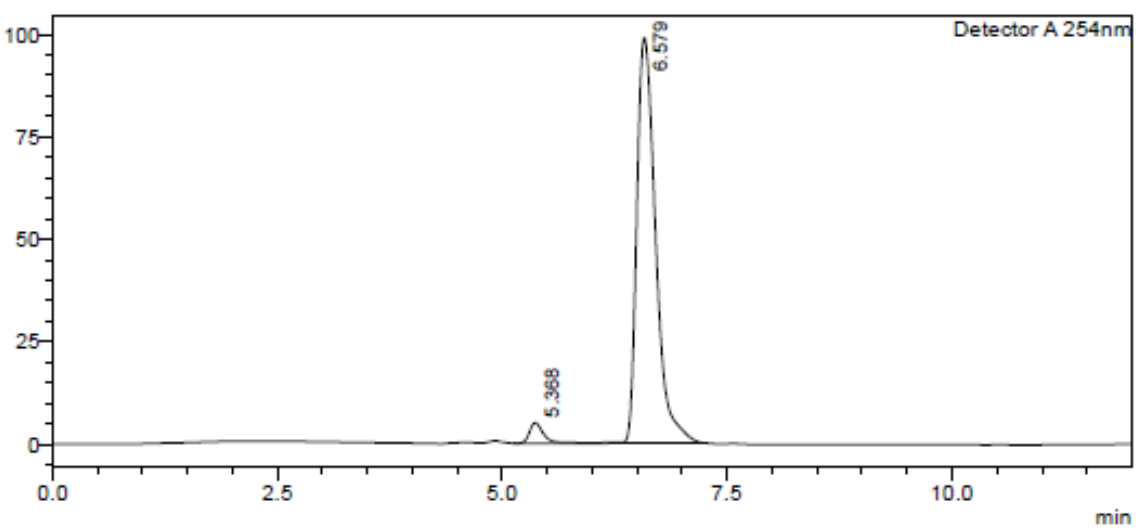

<Peak Table>

\begin{tabular}{|c|c|c|c|c|}
\hline \multicolumn{5}{|c|}{ Detector A $254 \mathrm{~nm}$} \\
\hline 1 & 5.368 & 59781 & 5085 & 4.004 \\
\hline 2 & 6.579 & 1433223 & 98868 & 95.996 \\
\hline ta & & 1493004 & 103953 & 100.000 \\
\hline
\end{tabular}

